\title{
Stören Kalziumpräparate die Wirkung von Kalziumantagonisten?
}

Frage: Beeinträchtigt die Einahme von Kalziumpräparaten die Wirkung von Kalzium-Antagonisten?

Prof. Dr. Jürgen C. Frölich, Hannover: Kalzium-Antagonisten sind Kalziumkanalblocker. Sie führen zu einer Entkopplung der elektrischen Erregung, zu einem verminderten Gefäßwiderstand im arteriellen System und Senkung der Nachlast.
Kalziumpräparate sind bei ernährungsoder malabsorptionsbedingtem Kalziummangel, bei Osteoporose und Hypoparathyreodismus indiziert. Intravenöses Kalzium wird als spezifisches Antidot bei einer Verapamil- oder Nifedipin-Überdosierung empfohlen. Laut der Fachinformation "Calciumgluconat 10\% B. Braun“ kann Kalzium die Wirkung von Kalzium-Antagonisten abschwächen. Ebenso wird vor der Einnahme von oralem Kalzium zusammen mit Verapamil oder anderen Kalziumkanalblockern gewarnt (MMI Pharmindex Plus 2012). Die Interaktion ist jedoch nur begrenzt untersucht. Die Kombination der Wirkstoffe sollte mit Vorsicht angewendet werden.

\section{Expertenrat Pharmazie,}

www.springermedizin.de
Frage: Ich behandle einen soweit gesunden Jungen (8 Jahre), bei dem nach einem Trauma vor zwei Monaten eine komplette Splenektomie durchgeführt wurde. Er wurde mit Pneumovax 23 geimpft, und hat bereits vor mehreren Jahren eine Meningokokkenimpfung $C$ erhalten. Macht eine weitere Impfung mit konjugiertem PneumokokkenimpfstoffSinn? Und ist die AntibiotikaProphylaxe (zwei Tagesdosen Amoxicillin, jeweils $20 \mathrm{mg} / \mathrm{kgKG}$ ) weiter angebracht? Wie reagiere ich auffieberhafte Infekte der Luftwege? Es muss ja nicht immer gleich ein Postsplenektomie-Syndrom sein.

Dr. Rüdiger Kardoff, Wesel: Menschen ohne funktionierende Milz sind vorrangig gefährdet durch septisch verlaufende Infektionen mit kapseltragenden Bakterien (v.a. Pneumokokken, aber auch Haemophilus influenzae und Meningokokken); diese drei Erreger verursachen hier über $80 \%$ der Sepsisfälle. Der Grad der Gefährdung hängt vom Alter bei Milzverlust ab und der bis dahin erworbenen Immunkompetenz, sowie vom Vorliegen einer immunologisch-hämatologischen Grunderkrankung. In diesem Fall unkritisch. Das Sepsisrisiko ist in der ersten Zeit nach dem
Milzverlust am höchsten, bleibt aber vermutlich lebenslang höher als in der Normalbevölkerung. Es wird angenommen, dass nicht wenige der Trauma-Patienten eine spontane Splenosis haben, d.h. eine hämatogene Absiedelung von versprengtem Milzgewebe, welches immunologische Funktionen aufnehmen kann.

Eine Penicillinprophylaxe wird für mindestens zwei Jahre nach Splenektomie empfohlen bzw. mindestens bis zum 6. Lebensjahr - noch länger nur wenn schon einmal eine invasive Pneumokokken-Infektion aufgetreten ist und beim Vorliegen einer hämatologisch-onkologischen Grunderkrankung. Eine 23-valente Pneumokokken-Immunisierung sollte laut STIKO alle 5 Jahre erfolgen. Fiebernde Patienten sollen frühzeitig mit einem gegen die o.a. Bakterien wirksamen BreitspektrumAntibiotikum behandelt werden, üblicherweise einem Cephalosporin der 2. bis 3 . Generation. Wann noch mit hinreichender Sicherheit ein Virusinfekt angenommen und auf eine antibiotische Therapie verzichtet werden kann, bleibt der ärztlichen Einzelfallentscheidung überlassen. Großzügiges Beiziehen von Laborbefunden (CRP, Blutbild) kann hilfreich sein.
Abschließend ist daran zu erinnern, dass Splenektomierte langfristig auch durch die Folgen der gestörten Clearance überalterter Erythrozyten gefährdet sind, nämlich thromboembolische Erkrankungen und pulmonale Hypertension.

\section{Expertenrat Pädiatrie,}

www.springermedizin.de

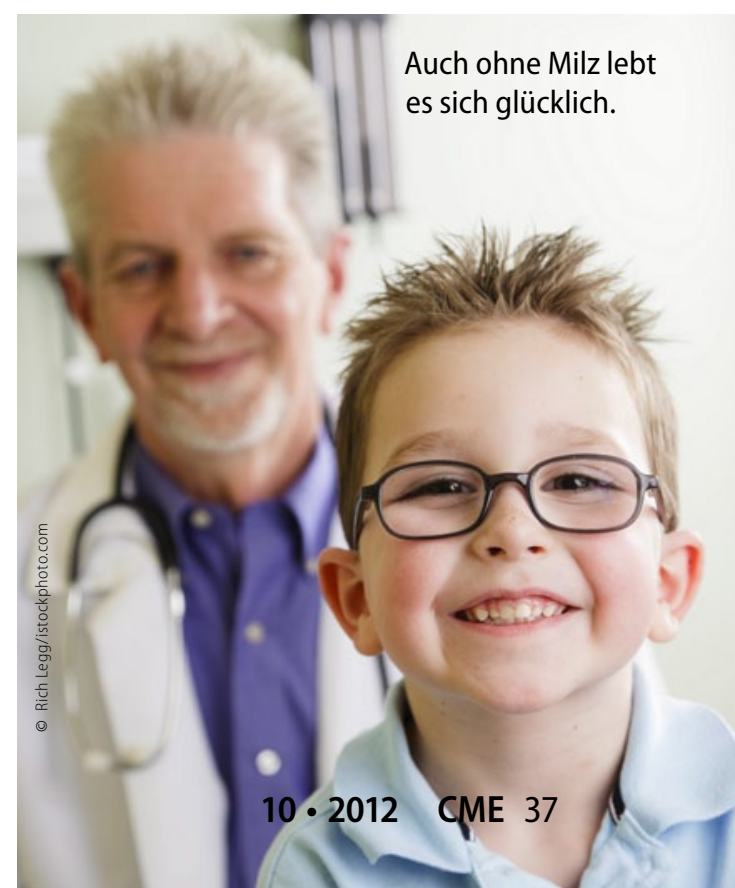

